09.6

\title{
Метод измерения периодов волноводных дифракционных оптических элементов
}

\author{
(C) С.С. Копенкин, В.В. Кесаев , А.Н. Путилин \\ Физический институт им. П.Н. Лебедева РАН, Москва, Россия \\ ๑ E-mail: vladimir_kesaev@mail.ru
}

Поступило в Редакцию 7 июля 2021 г.

В окончательной редакции 6 сентября 2021 г.

Принято к публикации 8 сентября 2021 г.

\begin{abstract}
Предложен оригинальный метод измерения периодов волноводных дифракционных оптических элементов. В отличие от стандартных методик предлагаемый метод позволяет проводить измерения для волноводных решеток дифракционных оптических элементов, когда дифрагировавшие порядки являются волноводными или световодными модами, распространяющимися в материале подложки. Метод обладает малой погрешностью и прост в техническом исполнении.
\end{abstract}

Ключевые слова: волноводные голограммы, дифракционные оптические элементы.

DOI: 10.21883/PJTF.2021.23.51785.18953

Одними из самых востребованных голографических оптических элементов (ГОЭ) в настоящее время являются волноводные голографические решетки, составляющие основу многих дисплеев дополненной реальности, например HoloLens, WaveOptics, DigiLens, Magic Leap и подобных $[1,2]$. Для оптимальной работы таких дисплеев необходимо обеспечить компенсацию хроматических искажений волноводных голограмм, а следовательно, контроль периодов и ориентации решеток крайне критичен. Однако дифрагированные лучи волноводных дифракционных оптических элементов (ДОЭ) или ГОЭ находятся в волноводе, и непосредственное измерение указанных параметров затруднено.

На рис. 1, $a$ приведена фотография типичного волноводного ГОЭ на стеклянном световоде (показатель преломления $n_{D} \approx 1.51$ ) толщиной $2 \mathrm{~mm}$ и протяженностью $200 \mathrm{~mm}$. Поверхностно-рельефная дифракционная решетка (ДР) небольшой апертуры выполнена голографическим способом на тонком слое фоторезиста. Период ее менее $0.5 \mu \mathrm{m}$, поэтому дифрагировавшие порядки для света с длиной волны $640 \mu \mathrm{m}$ распространяются исключительно в материале волновода. Для измерения периода ДР обычными способами [3,4] можно использовать дополнительные выводные призмы или изготовить скошенные полированные грани у световода (волновода), однако эти методы трудоемки и требуют точного учета геометрии и рефракционных свойств волновода. Использование методов микроскопии и микропрофилометрии позволяет быстро измерить период, при этом, однако, с небольшой точностью. На практике за счет поворота ДОЭ существует возможность изменения угла падения на ДР и вывода одного из дифрагировавших порядков из волноводного режима. В настоящей работе предлагается простая и эффективная методика для измерения параметров волноводных ДОЭ с помощью поворота ДР на угол наименьшего отклонения.
Метод заключается в соответствующей пространственной установке ДОЭ и измерении наименьшего угла отклонения $\theta^{\prime}$ первого дифракционного порядка $k= \pm 1$. Искомый период решетки $b$ для использованной длины волны (в вакууме) $\lambda$ находится по формуле

$$
b=\frac{\lambda}{2 \sin \frac{\theta^{\prime}}{2}} .
$$

При условии положительного знака у углов, отмеряемых от нормали к ДР по часовой стрелке, угол отклонения равен $\theta=\alpha-\varphi$ для пропускающей решетки и $\theta=\varphi-\alpha$ для отражательной, где $\alpha$ и $\varphi-$ угол падения и угол дифракции соответственно (рис. $1, b$ ). Оба эти угла связаны основным уравнением дифракционной решетки

$$
b(\sin \varphi-\sin \alpha)=k \lambda .
$$

При изменении угла падения $\alpha$ угол дифракции $\varphi$ также будет изменяться, при этом существует такое значение $\alpha=\varphi$, при котором угол отклонения окажется минимальным, т.е. $\theta=\theta^{\prime}$. Указанная установка ДР получила название автоколлимационной $[5,6]$. То обстоятельство, что при автоколлимационной установке ДР дифрагировавший порядок возвращается обратно к источнику, и позволяет относительно просто выполнить требуемое измерение периода $b$.

На рис. $1, b$ падающий под углом $\alpha$ луч $A$ дифрагирует в -1-й порядок под углом $\varphi$ в направлении вперед, от источника (луч $\left.B^{\prime}\right)$. Благодаря френелевскому отражению также возникает -1-й порядок в обратном направлении, к источнику излучения (луч $B$ ). Луч, дифрагировавший в $+1^{\prime}$-й порядок, превышающий критический угол (не обозначен), распространяется в материале подложки и представляет собой волноводную или световодную моду. Вторично попадая на ДР, он дифрагирует под тем же углом $\alpha$ в направлении к источнику. Этот луч, обозначенный буквой $C$, всегда будет 

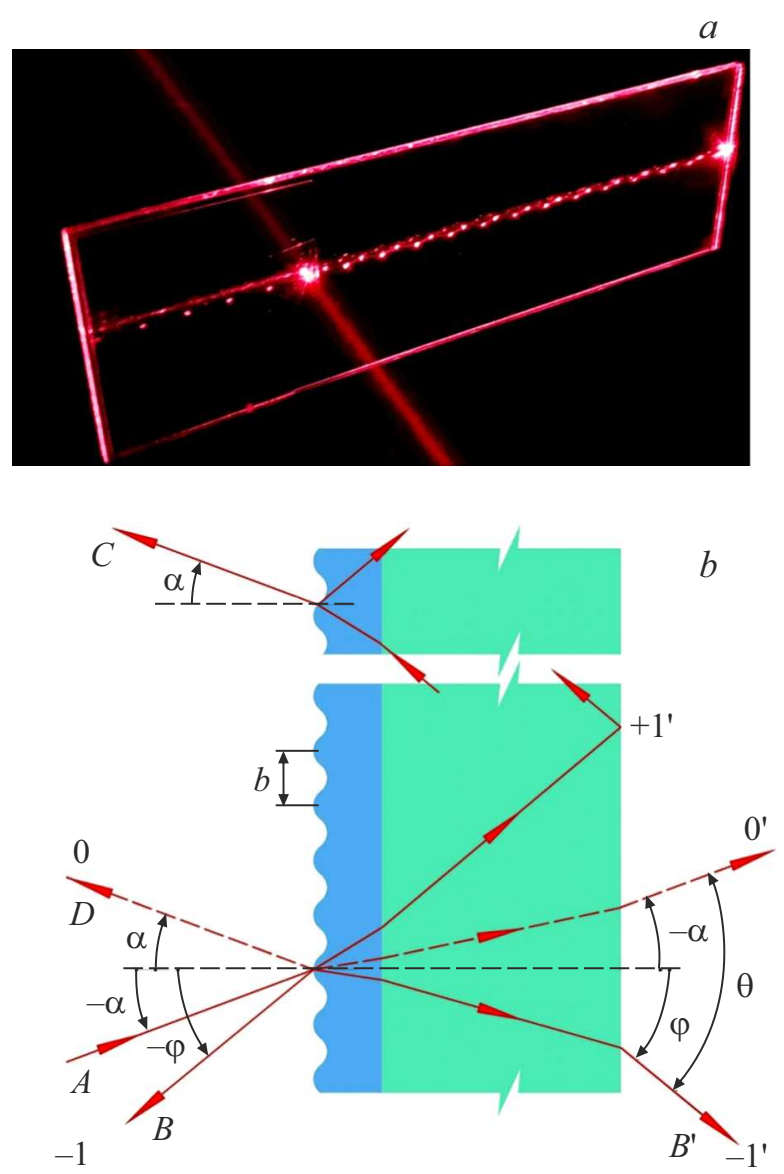

Рис. 1. $a-$ мода распространяющейся в материале подложки ГОЭ; $b$ - схема дифракции на волноводном ДОЭ.

отнесенным в пространстве на некоторое расстояние и параллельным зеркально отраженному лучу $D$.

Поворачивая ДР относительно нормали к рисунку, можно добиться равенства углов $\alpha=\varphi$, при этом луч $B$ вернется к источнику, а $\theta$ будет соответствовать наименьшему углу отклонения $\theta^{\prime}[7,8]$. Для того чтобы удостовериться в справедливости условия $\alpha=\varphi$, рассмотрим луч $B^{\prime}$, распространяющийся после дифракции вперед (рис. 1,b). Поскольку измерение углов выполняется в воздухе, показатель преломления материала волновода не влияет на результат, тогда, подставляя уравнение дифракции (2) для первого порядка в угол отклонения просветной ДР

$$
|\theta|=|\alpha-\varphi|
$$

дифференцируя по углу $\alpha$ и приравнивая получившееся выражение к нулю, получим условия экстремума

$$
\frac{d \theta}{d \alpha}=1-\frac{\cos \alpha}{\sqrt{1-\left(\frac{\lambda}{b}+\sin \alpha\right)^{2}}}=0 .
$$

Решая его, получим выражение для угла падения $\alpha$, соответствующего наименьшему углу отклонения $\theta^{\prime}$ :

$$
\alpha=\arccos \frac{\sqrt{4 b^{2}-\lambda^{2}}}{2 b} .
$$

Для доказательства $\alpha=\varphi$ достаточно, пользуясь (5), записать синус угла

$$
\sin \alpha=\sqrt{1-\frac{4 b^{2}-\lambda^{2}}{4 b^{2}}}=\frac{\lambda}{2 b},
$$

подставить его в формулу (2) и получить

$$
\sin \varphi=\frac{\lambda}{b}-\frac{\lambda}{2 b}=\frac{\lambda}{2 b}
$$

Для оценки погрешности метода обозначим половинный угол $\theta / 2=\beta \equiv(\alpha+\varphi) / 2$ в (1) и продифференцируем его по $\beta$. Отметим, что $\beta$ - это угол между нормалью к ДР и биссектрисой угла, образованной лучами $A$ и $B$, в автоколлимации $\beta=\alpha=\varphi=\theta^{\prime} / 2$. После замены дифференциалов конечными приращениями $d b \rightarrow \Delta$ и $d \beta \rightarrow \delta$ имеем

$$
\Delta=-\frac{\lambda \cos \beta}{2 \sin ^{2} \beta} \delta=-\frac{b}{\tan \beta} \delta .
$$

Здесь $\Delta$ - абсолютная ошибка периода $b$, а $\delta-$ погрешность в определении угла $\beta$. Знак минус означает, что при положительных ошибках $\delta$ рассчитываемое по формуле (1) значение периода будет уменьшаться. Разделив (8) на период $b$, получим выражение для относительной ошибки

$$
\varepsilon=\frac{\Delta}{b}=-\frac{\delta}{\tan \beta}
$$

Вид этой функции приведен на рис. 2. В области малых углов дифракции и при использовании стандартного поворотного столика с точностью $\pm 0.1^{\circ}$ предлагаемый метод не оптимален, тогда как при углах $\beta=\theta^{\prime} / 2 \geqslant 10^{\circ}$ погрешность уже не превышает $1 \%$ от периода. Для волноводных ГОЭ углы обычно превышают $30^{\circ}$, при этом при использовании стандартного поворотного столика с ошибкой $\pm 2^{\prime}$ метод обеспечивает точность измерения периода в пределах ошибки $\mp 1 \cdot 10^{-3}$.

На рис. 3 приводится экспериментальная зависимость половинного угла отклонения $\theta / 2$ от угла падения $\alpha$, выполненная для случая распространения луча в прямом направлении по схеме, представленной на рис. $1, b$ (луч $B^{\prime}$ ). Рисунок демонстрирует наличие экстремума угла $\theta=\theta^{\prime}$ при $\alpha=\varphi$. Для измерения этой зависимости ДОЭ устанавливался на гониометр, обеспечивающий точность $\pm 5^{\prime \prime}$, а в качестве источника света использовался лазер с длиной волны $\lambda=532 \mathrm{~nm}$. По измеренному углу наименьшего отклонения $\theta^{\prime}=72^{\circ} 03^{\prime} 08^{\prime \prime} \pm 5^{\prime \prime}$ рассчитанный по формуле (1) период составит $b_{\text {gon }}=452.26 \mp 0.02 \mathrm{~nm}$. В случае автоколлимационного измерения (с использованием луча $B$ на рис. $1, b)$ и поворотного столика с обеспеченной точностью $1^{\prime}$ измеренный угол составил величину $\beta=\theta / 2=36.033 \pm 0.017^{\circ}$, отвечающий ему период $b_{\text {auto }}=452.19 \mp 0.18 \mathrm{~nm}$. Отношение разницы полученных значений периодов к более точному значению составит $\frac{b_{\text {gon }}-b_{\text {auto }}}{b_{\text {gon }}} \approx 1.5 \cdot 10^{-4}$, что в абсолютном сравнении 


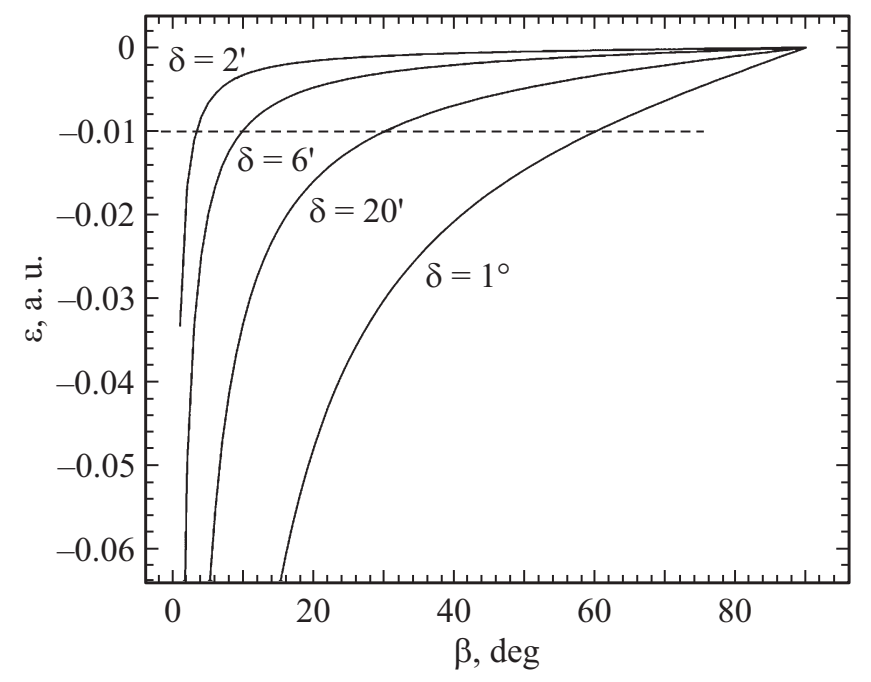

Рис. 2. Ошибка $\varepsilon=\Delta / b$ при определении значения периода в автоколлимационном режиме в зависимости от угла $\beta=\theta^{\prime} / 2$.

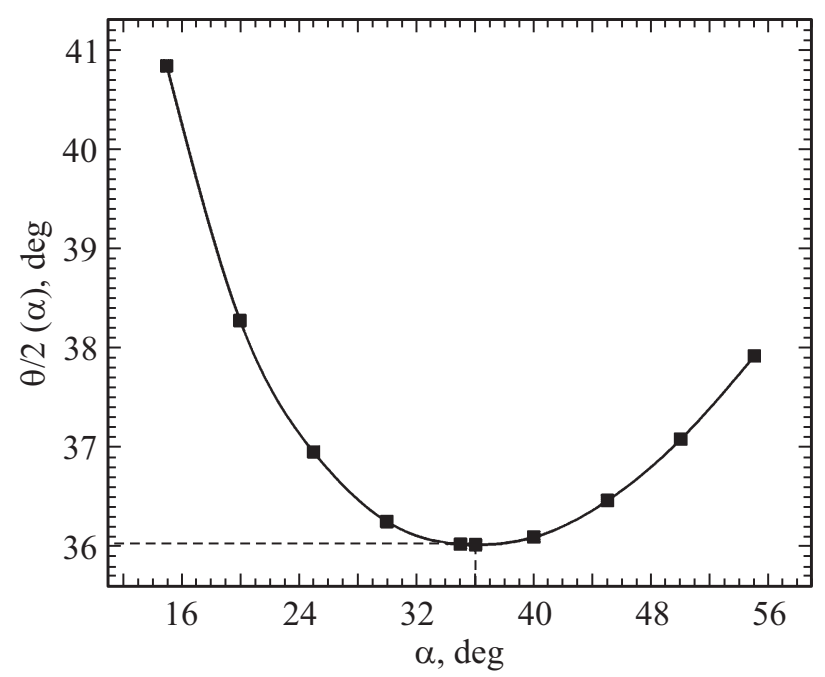

Рис. 3. Экспериментальная зависимость половинного угла отклонения $\theta / 2$ от угла падения $\alpha$ для решетки с периодом $b=452.26 \mp 0.02 \mathrm{~nm}$ и использованной длины волны монохроматического света $532 \mathrm{~nm}$.

не превышает $0.27 \mathrm{~nm}$ с учетом невыгодного сочетания погрешностей.

В заключение отметим практически важный случай „многослойного“ ГОЭ, используемого для формирования цветных изображений, в дисплеях дополненной реальности. Каждый слой ГОЭ содержит волновод и ДР, предназначенные для определенной длины волны (обычно красной, зеленой и синей). Измерение таких ДОЭ (ГОЭ) обычными способами затруднено, а предлагаемый метод позволяет относительно просто и с высокой на практике точностью определять периоды ДР для каждого слоя, не требуя при этом информации о материале и геометрии волновода. В настоящее время разработка дисплеев дополненной (смешанной) реальности идет в направлении усложнения оптических схем волноводных голограмм. Разработка новых методов исследования волноводных ДОЭ в этих условиях является актуальной и востребованной задачей.

\section{Финансирование работы}

Часть исследования выполнена В.В. Кесаевым за счет гранта Российского научного фонда № 20-71-10103.

\section{Конфликт интересов}

Авторы заявляют, что у них нет конфликта интересов.

\section{Список литературы}

[1] B.C. Kress, Proc. SPIE, 11062, 110620J (2019). DOI: $10.1117 / 12.2527680$

[2] J. Chen, W. Cranton, M. Fihn, Handbook of visual display technology (Springer International Publ., Switzerland, 2016), p. 205. DOI: 10.1007/978-3-319-14346-0

[3] D. Apostol, P.C. Logofatu, S. Florea, V. Damian, I. Iordache, M. Bojan, J. Optoelectron. Adv. Mater., 10 (2), 352 (2008).

[4] J.E. Harvey, R.N. Pfisterer, Opt. Eng., 58 (8), 087105 (2019). DOI: $10.1117 / 1 . O E .58 .8 .087105$

[5] К.И. Тарасов, Спектральные приборы (Машиностроение, Л., 1968), c. 80.

[6] И.В. Пейсахсон, Оптика спектральных приборов (Машиностроение, Л., 1975), с. 54.

[7] Q. Wang, Optik, 124 (23), 6349 (2013). DOI: $10.1016 /$ j.jileo.2013.05.052

[8] P. Mohazzabi, D.J. Mattson, J.A. Ponce, Jr, J. Appl. Math. Phys., 7 (8), 1870 (2019). DOI: 10.4236/jamp.2019.78128 